\title{
Correction to: Downscaling Images with Trends Using Multiple-Point Statistics Simulation: An Application to Digital Elevation Models
}

\author{
Luiz Gustavo $\operatorname{Rasera}^{1}$ (D) Mathieu Gravey ${ }^{1}$ (D) \\ Stuart N. Lane ${ }^{1}$ (D) Gregoire Mariethoz ${ }^{1}($ ID
}

Published online: 10 October 2019

(C) International Association for Mathematical Geosciences 2019

\section{Correction to: Math Geosci https://doi.org/10.1007/s11004-019-09818-4}

The original version of this article unfortunately contained mistakes in Eqs. 9, 10, 12 and 13 .

The correct versions are

$$
\begin{gathered}
D\left(d_{V}\left(\mathbf{u}_{i}\right), d_{V}^{(t)}(\mathbf{u})\right)=\left(\sum_{j=0}^{n_{V}} \lambda\left(\mathbf{h}_{j}\right)\left[r_{V}\left(\mathbf{u}_{i}+\mathbf{h}_{j}\right)-r_{V}^{(t)}\left(\mathbf{u}+\mathbf{h}_{j}\right)\right]^{2}\right)^{1 / 2}, \\
D\left(d_{v}\left(\mathbf{u}_{i}\right), d_{v}^{(t)}(\mathbf{u})\right)=\left(\sum_{j=1}^{n_{v}\left(\mathbf{u}_{i}\right)} \lambda\left(\mathbf{h}_{j}\right)\left[r_{v}^{(s)}\left(\mathbf{u}_{i}+\mathbf{h}_{j}\right)-r_{v}^{(t)}\left(\mathbf{u}+\mathbf{h}_{j}\right)\right]^{2}\right)^{1 / 2} \\
\operatorname{Pr}\left\{\mathbf{R}_{v}\left(\mathbf{u}_{i}\right)=\mathbf{r}_{v} \mid \Omega_{i-1}\right\} \approx \operatorname{Pr}\left\{\mathbf{R}_{v}\left(\mathbf{u}_{i}\right)=\mathbf{r}_{v}^{(t)}\left(\mathbf{u}_{k}\right) \mid d_{V}\left(\mathbf{u}_{i}\right), d_{v}\left(\mathbf{u}_{i}\right)\right\} \\
\propto \operatorname{Pr}\left\{\mathbf{R}_{v}\left(\mathbf{u}_{i}\right)=\mathbf{r}_{v}^{(t)}\left(\mathbf{u}_{k}\right) \mid d_{V}\left(\mathbf{u}_{i}\right)\right\}^{1-\alpha_{i}} \cdot \operatorname{Pr}\left\{\mathbf{R}_{v}\left(\mathbf{u}_{i}\right)=\mathbf{r}_{v}^{(t)}\left(\mathbf{u}_{k}\right) \mid d_{v}\left(\mathbf{u}_{i}\right)\right\}^{\alpha_{i}} \\
\lambda\left(\mathbf{h}_{j}\right)=\frac{1}{2 \pi \sigma^{2} \beta} \exp \left(-\frac{\left\|\mathbf{h}_{j}-\mathbf{h}_{0}\right\|_{2}^{2}}{2 \sigma^{2}}\right)
\end{gathered}
$$

Please note that the correct versions of the equations were used in the computations, therefore, the results remain unchanged.

The original article can be found online at https://doi.org/10.1007/s11004-019-09818-4.

$凶 \quad$ Luiz Gustavo Rasera

luizgustavo.rasera@unil.ch

1 Institute of Earth Surface Dynamics, Faculty of Geosciences and Environment, University of Lausanne, 1015 Lausanne, Switzerland 